\title{
The West and the Rest? Zur postkolonialen Kritik an Global Citizenship Education
}

\begin{abstract}
Zusammenfassung
In diesem Beitrag verteidige ich eine universalistische Konzeption der Global Citizenship Education (GCE) gegen eine der zentralen Kritiken, die unter anderem im Rahmen von postkolonialen Theorieansätzen vorgebracht wurde. Diese Kritik moniert, dass GCE von genuin, westlichen Werten' ausgeht (z. B. in der Form einer Menschenrechtsbildung und -erziehung oder von Konzeptionen der Rationalität oder des Selbst), die weder universell akzeptiert seien, noch universelle Geltung beanspruchen könnten und daher illegitimer Weise Angehörigen nicht westlicher Kulturen und Gesellschaften pädagogisch aufoktroyiert würden. Gemäß dieser Kritik repräsentiert GCE nur eine weitere Variante von pädagogisch ambitionierten Rechtfertigungen eines hegemonialen und ungerechten globalen Regimes in der Tradition des westlichen Kolonialismus und Imperialismus.
\end{abstract}

Schlüsselworte: Global Citizenship Education, postkoloniale Theorie, Universalismus

\begin{abstract}
This contribution develops a defense of a universalist conception of Global Citizenship Education (GCE) against one of its most central critiques. This critique argues that GCE is based on genuinely 'Western values' (e. g., in the form of a conception of human rights or conceptions of rationality or the self), which are neither universally accepted nor universally valid and therefore unjustly forced on members of non-Western cultures and societies. GCE, according to this critique, is assumed to be another version of the educational justification of a hegemonic and unjust global regime in the tradition of Western colonialism and imperialism.
\end{abstract}

Keywords: Global Citizenship Education, postcolonial theory, universalism

\section{Einleitung}

$\mathrm{Zu}$ den klassischen Kritiken an Konzeptionen einer weltbürgerlichen Erziehung und Bildung (in der internationalen Debatte: Global Citizenship Education; kurz: GCE) gehört der Vorwurf, dass sie auf eine arbiträre Extrapolation genuin ,westlicher Werte' auf andere gesellschaftliche und kulturelle Kontexte hinauslaufen und als pseudo-universalistischer Ausdruck neokolonialer und -imperialer pädagogischer und politischer Ambitionen zu kritisieren seien. Eine gewisse Plausibilität erhält diese Kritik durch das historische Faktum, dass sowohl Kolonialismus als auch Imperialismus in vielen Fällen mit Rekurs auf pädagogische und paternalistische Rechtfertigungsmuster begründet wurden. ${ }^{1}$ Den Kolonisierten wurde z. B. von liberalen Denkern wie James und John Stuart Mill unterstellt, unfähig zu sein, sich selbst zu bestimmen. Sie seien deshalb auch zu ihrem eigenen Wohl auf die advokatorisch-benevolente Repräsentation durch Kolonisatoren angewiesen. Wie Kinder, so die gängige paternalistische Metaphorik, müssten sie erzogen, gebildet und auch diszipliniert werden, um am Ende eines Tages auch als autonome und selbstbestimmungsfähige Menschen ihr Leben führen zu können (z. B. McCarthy, 2009; Arneil, 2012). ,Westliche Bildung' und ,westliche Kultur rechtfertigen die Superiorität des kolonialen und imperialen Projekts. Diese Form der Rechtfertigung und die entsprechende metaphorische Rahmung des Verhältnisses von Kolonisatoren und Kolonisierten wurde bis ins 20. Jahrhundert hinein genutzt und ist auch in der heutigen postkolonialen Welt noch von Relevanz (Hobson, 2017; Barnett, 2017; Liebel, 2017). Superioritätsansprüche werden heute jedoch häufig weniger direkt durch rassistische Vorurteile gerechtfertigt, sondern eher mit Hilfe der Konstruktion von kulturellen, epistemischen und pädagogischen Differenzen. Die richtige Form der Erziehung und der Bildung und die richtige Kindheit sind in dieser politischen Denkökonomie westliche Formen der Erziehung und Bildung und westliche Vorstellungen von Kindheit. Kolonialismus und Imperialismus ${ }^{2}$ verstanden sich entsprechend in vielen Fällen auch als in weitem Sinne genuin 
pädagogische Projekte und nutzten für ihre Zielvorgaben (Formen der Beschulung, Schulorganisation etc.) und deren Rechtfertigung tradierte pädagogische Metaphoriken.

Man kann und sollte also durchaus skeptisch sein gegenüber kontemporären Konzeptionen der Erziehung und Bildung, die, wie Global Citizenship Education (GCE), für sich in Anspruch nehmen, das Ensemble von Fähigkeiten, Dispositionen und Wissen zu vermitteln, damit Kinder, Weltbürgerinnen' bzw. ,Weltbürger' werden können. Die Vermutung liegt nahe, dass im Namen von GCE eine neue Variante des westlichen pädagogischen Imperialismus gefördert wird, die dem Rest der Welt aufoktroyiert werden soll.

In diesem Beitrag verteidige ich eine universalistische Konzeption der Global Citizenship Education gegen eine der zentralen Kritiken, die unter anderem im Rahmen von postkolonialen Theorieansätzen ${ }^{3}$ vorgebracht wurde. Diese Kritik moniert, dass GCE von genuin, westlichen Werten' ausgeht (z. B. in der Form einer Menschenrechtsbildung und -erziehung oder von Konzeptionen der Rationalität oder des Selbst), die weder universell akzeptiert sind, noch universelle Geltung beanspruchen können und daher Angehörigen nicht westlicher Kulturen und Gesellschaften illegitimer Weise pädagogisch aufgezwungen werden. Gemäß dieser Kritik stellt GCE nur eine weitere Variante von pädagogisch ambitionierten Rechtfertigungen eines hegemonialen und ungerechten globalen Regimes dar, die in der Tradition des westlichen Kolonialismus stehen. Meine Kritik dieser Kritik geht davon aus, dass postkoloniale Theorieperspektiven unbedingt notwendig und relevant sind für eine (selbst-)kritische Konzeption der GCE. Zugleich kann und sollte jedoch, so meine Argumentation, eine solche Konzeption auf die Begründung universalistischer Prinzipien und universaler Geltungsansprüche nicht verzichten.

\section{Global Citizenship Education: Grundzüge einer universalistischen Konzeption}

GCE kann im weitesten Sinne verstanden werden als eine pädagogische Form der Reaktion auf und des Umgangs mit unterschiedlichen moralischen, ökonomischen, kulturellen, spirituellen und ökologischen Dimensionen von Globalisierung. Der Terminus GCE ist hoch umstritten. Als Regenschirmbegriff (umbrella term $)^{4}$ umfasst er, unter anderem, Ansätze der politischen Bildung, der Demokratieerziehung und der Menschenrechtsbildung, der Friedenserziehung, der Bildung für nachhaltige Entwicklung und des inter- und transkulturellen Lernens. Unterschiedliche Konzeptionen von GCE können diese oder auch andere Elemente jeweils unterschiedlich gewichten und interpretieren (Oxley \& Morris, 2013). In vielen Fällen gibt es zudem Überschneidungen zwischen eher national orientierten Formen der Demokratieerziehung in pluralistischen Gesellschaften und transnational orientierten Konzeptionen der GCE (de Ruyter \& Spiecker, 2008). GCE wird in unterschiedlichen Varianten propagiert von Regierungen, internationalen Organisationen (UNESCO etc.) in formalen (Schulen, Lehrerausbildung etc.) und eher informellen Settings (Jugendarbeit, street work). Wichtig ist es in diesem Zusammenhang zu sehen, dass GCE üblicherweise als eine auf regulativen Idealen basierende pädagogische, ethische, politische, rechtliche und soziokulturelle Leitidee verstanden wird und werden muss, da es so etwas wie eine globale Staatsbürgerschaft streng genommen heute noch nicht gibt (insbesondere ohne hinreichend durchsetzungsfähige supranationale Organisationen oder einen Weltstaat, der z. B. die Menschenrechte überall auf dem Planeten durchsetzen könnte).

Die Konzeption von GCE, die im Folgenden diskutiert und verteidigt werden soll, basiert auf der bildungstheoretischen Idee einer Verknüpfung des Lokalen und des Alltäglichen mit dem Globalen und dem Universalen (Gaudelli, 2016). Typische Themen von GCE können z. B. anhand von alltäglichen Konsumgütern wie einer Jeans oder auch einer Tomate (Hendriks, 2018) erläutert und diskutiert werden. So lässt sich z. B. fragen, unter welchen Bedingungen die Tomate produziert wurde und wie die Jeans in den Discounter um die Ecke gekommen ist. Durch Nachdenken über die internationalen Lieferketten und die ungleichen Arbeitsbedingungen, welche in der Produktion von Tomaten relevant sind, werden die Adressaten aufgefordert, ihre ortsgebundene alltägliche Perspektive zu transzendieren und die globale Dimension ihrer Existenz mit der lokalen Dimension reflexiv zu verknüpfen. Eine kritische Reflexion von Alltagsüberzeugungen im Lichte von Prinzipien höherer Ordnung und die Transzendierung und Transformation dieser Überzeugungen über die Vermittlung von Wissen und Bewusstsein für globale Interdependenzen sind ein zentraler Aspekt von GCE. Nach Gaudelli (2016, S. 163) zielt GCE als eine Form der Transzendierung des Alltäglichen auf die Kultivierung und Förderung von intelligenten Gewohnheiten ab, deren wichtigstes Charakteristikum er wie folgt umschreibt: „The habit that GCE aims to encourage is simply mindfulness about how the world is present in all material and relational interactions, a habitual way of thinking that actively works away from the way we tend to see ourselves in the world and towards the way that we need to perceive ourselves: from isolated to integrated, disconnected to interconnected and separated to inseparable" (ebd.).

Als eine Transfiguration und Transformation von Selbst-, Welt und Sozialverhältnissen in unterschiedlichen Dimensionen (ethisch, politisch, ökonomisch, ästhetisch etc.), über welche potentiell unlimitierte Verknüpfungen zwischen Alltäglichem und Universellen gefasst und integriert werden, stellt GCE ein regulatives Bildungs- und Erziehungsideal dar. Als eine Form der Allgemein- und Grundbildung 5 in und für eine zunehmend global integrierte Welt umfasst GCE eine Reihe von spezifischeren Erziehungs- und Bildungszielen, die von der Leitidee ausgehen, das Lokale und das Globale miteinander zu verbinden (und vice versa), durch die Förderung von:

1. einem Bewusstsein für und kritischer Reflektion von globalen ökonomischen, kulturellen und politischen Interdependenzen, der historischen Genese von globalen Ungleichheiten (z. B. über Kolonial- und Globalgeschichte) und der Kritik und Kontextualisierung von fragwürdigen universalistischen Geltungsansprüchen (z. B. einseitige Konzeptionen der ,Entwicklung' und des ,Fortschritts');

2. der Differenzierung und Erweiterung von kognitiven und ethischen Referenz- und Bedeutungshorizonten und der Reflexion darüber, wie die eigene Position und Ortsgebundenheit die Art und Weise beeinflusst, wie man die 
Welt sieht, um so personale Autonomie (d. h. in zentraler Hinsicht die Fähigkeit zu kritischer Selbstreflektion) und politische Autonomie (d. h. vor allem die Fähigkeit zu informierter Partizipation in einer entstehenden globalen Öffentlichkeit) ${ }^{6}$ zu ermöglichen;

3. von Wissen über und Verständnis für die im stetigen Wandel begriffenen ,Kulturen', Traditionen, lokalen Perspektiven, und Entwicklungen der internationalen Politik auch im Gefüge supranationaler Institutionen;

4. von kosmopolitischen und liberal-demokratischen Einstellungen und Tugenden, wie z. B. ,open-mindedness', Akzeptanz oder (zumindest) Toleranz für unterschiedliche Aspekte von ,Diversität', die für transnationale Formen der Partizipation und Kooperation notwendig sind;

5. von Verständnis und Akzeptanz basaler, universell gültiger rechtlicher, moralischer und politischer Prinzipien, insbesondere der Menschenrechte (z. B. Brumlik, 2016);

6. sowie von universellen Formen der Identifizierung und der Identität als Weltbürger/innen, welche partikularere Identitäten transzendieren, jedoch nicht notwendigweise mit diesen inkompatibel sind (z. B. Formen der europäischen Identität, Fukuyama, 2018).

Global citizenship education als pädagogisches Projekt umfasst moralische, politische, rechtliche, kulturelle, ökonomische, spirituelle und ökologische Dimensionen. Es ist zudem sinnvoll davon auszugehen, dass GCE weder notwendig an ein spezifisches Unterrichtsfach (z. B. in öffentlichen Schulen) gebunden ist und entsprechend durch sehr unterschiedliche Mittel in der Praxis umgesetzt werden kann (z. B. durch eine Erweiterung des Kanons), noch auf ein spezifisches Set von Praktiken reduziert werden kann (Sant et al., 2018, S. 27). Dies hat zugleich jedoch nicht die relativistische Implikation, dass ein ,korrekter' und ,richtiger' Ansatz von GCE sich nicht begründen und praktizieren ließe (ebd.). Natürlich lassen sich angemessenere und weniger angemessene Ansätze von GCE unterscheiden, so wie auch die Werte und Prinzipien, auf denen unterschiedliche Konzeptionen basieren, als mehr oder weniger gültig und verbindlich ausgewiesen werden können. Die von mir im Folgenden vertretene Konzeption von GCE weist eine starke kosmopolitische Orientierung auf in dem Sinne, dass sie auf vier unterschiedlichen Typen von Universalismus aufbaut:

1. Moralischer und rechtlicher Universalismus: GCE basiert wesentlich auf einer Konzeption der Menschenrechtsbildung und auf der Anerkennung der normativen Geltung von Menschenrechten, so wie sie in der, Universal Declaration of Human Rights' und in der, Convention on the Rights of the Child' festgelegt wurden (Gaudelli, 2016).

2. Pädagogischer Universalismus: GCE basiert darüber hinaus auf einer Konzeption von Bildung und Erziehung, die davon ausgeht, dass das Ideal der kritischen Reflexion, Transzendierung und Transformation von Selbst-, Welt- und Sozialverhältnissen einen universell gültigen pädagogischen Standard darstellt. Eine solche autonomiebasierte Konzeption ist inkompatibel mit stark partikularistischen und kommunitaristischen Konzeptionen der Erziehung und Bildung.
3. Politischer Universalismus: GCE fußt auf einer Konzeption der transnationalen Demokratieerziehung und politischen Bildung (Culp, 2018/2019), die davon ausgeht, dass demokratische Selbstbestimmung ein fundamentales Menschenrecht ist, personale und politische Autonomie koordinierte und universell gültige pädagogische und politische Ziele sind und eine zunehmende politische und nicht nur funktionale Integration (jenseits des Nationalstaats in Richtung einer globalen Demokratie) längerfristig für die Einrichtung einer gerechteren globalen Ordnung notwendig sein wird (hierzu: Held, 1995). Diese Konzeption ist entsprechend ausgerichtet auf die Identifikation mit einer in Entstehung begriffenen transnationalen politischen Gemeinschaft und nicht kompatibel mit Formen der Demokratieerziehung, die alleine auf den nationalen und lokalen Kontext bezogen sind.

4. Epistemologischer Universalismus: GCE geht von der Annahme aus, dass eine Pluralität von Perspektiven, Wissensformen und Formen des Wissenserwerbs kompatibel und in eine universelle und transkulturelle Epistemologie integrierbar ist. Eine solche Epistemologie geht von einer evolutionär entstandenen und in Grenzen veränderbaren menschlichen Natur und der Existenz einer geteilten bzw. zumindest (mit-)teilbaren objektiven Realität aus, die prinzipiell für jeden Menschen zugänglich ist. Dieser epistemologische Universalismus ist inkompatibel mit radikalen Versionen des Konstruktivismus, Relativismus und Antirealismus und epistemologischen Ansätzen, die davon ausgehen, Epistemologie sei ein essentiell politisches Unternehmen, welches notwendig und vollends durch seine Einbettung in Machtstrukturen und -diskurse determiniert ist. Er geht dagegen von der generellen Kompatibilität menschlicher Erfahrung aus (Conrad, 2016, S. 198) und ist somit nicht vereinbar mit Annahmen einer ,radikalen epistemischen Differenz $z^{\prime}$, die davon ausgeht, dass es aus ,kulturellen' oder anderen Gründen grundsätzlich unmöglich sei ,den Anderen`zu verstehen. Diese Unvereinbarkeit gilt auch für Konzeptionen, die einen radikal unterschiedlichen epistemischen ,Anderen ' postulieren und annehmen, dass sich die epistemischen Fähigkeiten von Menschen je nach Traditionen oder Kulturen, in denen sie aufgewachsen oder zu Hause sind, so radikal unterscheiden, dass Verständigungen verunmöglicht werden. ${ }^{7}$

Zusammengenommen liefern diese vier Formen des Universalismus (welche selbstverständlich alle in der Debatte kontrovers diskutiert werden) einen Rahmen, um die normativen Ausgangsprämissen und Leitorientierungen von GCE als pädagogischem Projekt zu klären. Als universalistisches, moralisches und rechtliches Projekt geht GCE von einer Konzeption globaler Bildungsgerechtigkeit aus, welche annimmt, dass jedes Kind das Recht hat, die Fähigkeiten, Kompetenzen und Dispositionen zu entwickeln und zu kultivieren, die notwendig sind, um eine Weltbürgerin bzw. ein Weltbürger im hier dargelegten Sinne zu werden. Als eine pädagogische Konzeption muss sie sich jedoch mit Bezug auf die exakte Interpretation der relevanten Prinzipien, die genaue Bestimmung der transnationalen Verantwortlichkeiten, die mit diesen verbunden sind, mit Blick auf das Verhältnis zwischen lokalen und globalen Loyalitäten 
ein Stück weit in Zurückhaltung üben und offen bleiben. Eine Konzeption von GCE, die hier keinen Raum für Interpretation und Diskussion ließe, müsste sich nicht nur mit der Kritik des westlichen Imperialismus, sondern auch mit der Kritik der Indoktrination auseinandersetzen. ${ }^{8} \mathrm{Zu}$ den zentralen rechtfertigungstheoretischen Problemen und Herausforderungen, die die genannten Universalismusformen bearbeiten müssen, gehören die folgenden: erstens, die Frage woher das Universelle kommt, wo seine Ursprünge und Entstehungskontexte sind und ob es jeweils universell gültig ist oder nur im Kontext einer bestimmten Tradition; zweitens die eng damit verbundene Frage, wie das Universelle jeweils im Kontext einer hochgradig pluralistischen Welt gerechtfertigt werden kann; und drittens wie das Universelle verbunden, eingebettet und auf das Partikulare und Lokale, ohne das Partikulare im Namen des Universellen zu unterdrücken, angewendet werden kann. Im Folgenden werde ich weder eine vollständige Rechtfertigung noch eine vollständige Verteidigung dieser vier Formen von Universalismus gegen alle denkbaren Einwände und Gegenargumente liefern können. Mein Argumentationsziel ist bescheidener, in dem Sinne, dass ich zeigen will, dass eine universalistische Konzeption der GCE gegen eine der wichtigsten, partikularistischen Kritiken, die unter anderem von der postkolonialen Theorie vorgebracht werden, verteidigt werden kann.

\section{The West and the Rest: Zur Kritik von Global Citizenship Education}

Im Kontext kolonialer Regime wurde eine, Westliche Bildung in der Regel als Ausdruck der vermeintlichen Überlegenheit der Kolonialmächte angesehen und diente zugleich als ein wirkmächtiges Instrument kolonialer Dominanz (z. B. Reinhard, 2018). Eine der wichtigsten Kritiken an GCE geht davon aus, dass GCE auf Reproduktion und Perpetuierung von pädagogischen Rechtfertigungen des Kolonialismus und Imperialismus hinausläuft, so wie sie aus dem 19ten und 20ten Jahrhundert bekannt sind. Der ,Diskurs' der GCE, so die Kritik, sei nicht zu trennen von welt-gesellschaftlichen Hierarchien und Ungleichheiten. Der Verweis auf,Bildung' diente in diesem Kontext zur Rechtfertigung unterschiedlicher Formen des Neo-Imperialismus (kulturell, ethisch, moralisch, politisch, ökonomisch etc.). Diese Kritik geht in unterschiedlichen Spielarten davon aus, dass GCE ein genuin westliches Projekt sei, entstanden und gestützt durch eine arbiträre Extrapolation von partikularen westlichen Werten und entsprechenden Erziehungs- und Bildungsidealen (z. B. personale Autonomie, Individualismus, Menschenrechte, Demokratie, Liberalismus; vgl. Mignolo, 2009; Tully, 2008; Lee, 2014; Mouffe, 2014; Dill, 2015; Wohlfahrt, 2018), Konzeptionen der Rationalität (etwa: instrumentelle Rationalität) und des Selbst (z. B. das ,punktförmige‘: Taylor, 1989 oder , unencumbered self: Sandel, 1998) auf andere soziale und kulturelle Kontexte. Das Projekt sei deshalb als pseudo-universalistisch, neo-imperialistisch und intolerant gegenüber anderen Traditionen zu kritisieren und abzulehnen. Aus der Perspektive dieser Kritik ist GCE Ausdruck der „latest version of the long march of the Enlightenment ideal of the liberated, tolerant self in Western liberal democracies" (Dill, 2015, S. 100) und eines naiven Universalismus, der die eigenen eurozentristischen Standards auf die Welt projiziert und damit in mehr oder weniger direkter Kontinuität zu der zutiefst ungerechten Politik kolonialer Regime steht.

Ich argumentiere im Folgenden, dass eine universalistische Konzeption von GCE keineswegs inkompatibel ist mit moderaten Formen der postkolonialen Kritik des Missbrauchs verschiedener Formen des Universalismus, sondern dass diese Kritik als wichtiger Bestandteil von GCE zu berücksichtigen ist. Die Kritik von GCE als Ausdruck von genuin westlichen Werten unterschätzt jedoch die eigene Angewiesenheit auf eben diese Wertbezüge als Begründungsressourcen und die Dynamik der globalen Verbreitung von normativen Idealen; sie überschätzt den Konsens bezüglich dieser Werte innerhalb der ,Westlichen Tradition' (vgl. z. B. die Kontroversen zwischen Liberalismus und Kommunitarismus oder zwischen politischem Liberalismus und liberalem Perfektionismus) und operiert entsprechend mit hochgradig vereinfachten und essentialistischen Annahmen über die, Westliche Tradition“. „Western thought is diverse and no longer neatly distinguishable from alternative traditions. There are no easy boundaries between the 'West' and 'the rest'" (Enslin \& Horsthemke, 2015, S. 1168). Während dieses Problem natürlich auch im Rahmen von einigen postkolonialen Theorieansätzen gesehen wurde, die sich in zentraler Hinsicht der kritischen Dekonstruktion entsprechender Dichotomien (,The West and the Rest'; vgl. die klassische Kritik von: Hall, 1992) verschrieben haben und dabei selbst auf die argumentativen und theoretischen Ressourcen und Ideen, westlicher' und, nicht westlicher' Denker und Traditionen zurückgreifen (Loomba, 2015), kann die eher simple und exklusiv-geographisch konzipierte Konstruktion des, Westens', der, Westlichen Tradition' oder auch, Westlicher Werte', die dann z. B. dem, Osten' oder dem, Orient' gegenübergestellt werden (vgl. hierzu die Kritiken von: Sen, 2006; Weidner, 2018; Appiah, 2018), auch historisch erklärt werden.

Mishra stellt hierzu fest: „This meant that the greater the scale of humiliation by the West, the more intense was the desire to posit an idealized image of the East. Nevertheless, Tagore and Liang Qichao represented a strong early trend, still visible today, of Asian intellectuals defining Western modes of politics, economics, science and culture as inhumanly utilitarian" (Mishra, 2013, S. 254). Auch wenn dies historisch und politisch verständlich war, basieren solche reduktionistischen Konstruktionen des, Westens' und die damit häufig verbundenen Äquivalenzunterstellungen (Universalismus = Westen = Imperialismus etc.; vgl. die Kritik hieran von: Dübgen, 2017) auf geradezu spektakulären Vereinfachungen, die weder in kontemporären philosophischen und wissenschaftlichen Debatten über GCE noch in praktischen Programmen und Konzeptionen von GCE eine Rolle spielen sollten. ${ }^{9}$ Während diese Kritiken also den Konsens in der, Westlichen Tradition ' überschätzen, unterschätzen sie zugleich die Dynamik des globalen Austauschs von Ideen. Demokratie (Sen, 2005) oder Toleranz (Nederman, 2012), zum Beispiel, sind mit Sicherheit keine exklusiv westlichen Ideen und es ist gerade ein zentrales Desiderat von GCE, ein Bewusstsein zu fördern für die Risiken einseitiger Konstruktionen von ,Traditionen' und für die historischen Verbindungslinien und Verwobenheiten zwischen unterschiedlichen Traditionen. Postkoloniale Kritiken ähneln häufig auch mehr oder weniger innovativen Reproduktionen 
und Adaptionen von eher traditionellen Formen der ,westlichen'Selbstkritik, so wie sie z. B. von der kritischen Theorie und verschiedenen Versionen des Kommunitarismus vorgetragen wurden (z. B. Sandels Kritik des, unencumbered self, oder Kritiken der ,instrumentellen Rationalität' von Horkheimer, Adorno und anderen, deren Positionen wiederum zurecht ebenfalls als eurozentristisch kritisiert wurden; vgl. Said, 1994; Allen; 2017). Dies stellt ja auch kein Problem dar, es wird nur dann zum Problem, wenn man philosophiehistorische und wissenschaftsgeschichtliche Kontexte ausblendet, für die eigene Sichtweise als Begründungsstütze Authentizität beansprucht und das offensichtliche Faktum nicht berücksichtigt, dass z. B. Demokratie und Menschenrechte auch im , Westen' nicht universell akzeptierte Werte darstellen. Als universelle Werte ,gehören' sie ohnehin zu keiner partikularen und exklusiven ,Tradition' oder einem bestimmten geographisch fixierten Ort: „Or, to put it another way, what made Enlightenment ideas truly universal was that they became weapons in the hands of those who fought Western imperialism (...). The ideals of liberty, equality, democracy and rights are not specific to the West. They are applicable to Haitans, to Indians and to South Africans" (Malik, 2014, S. 332). Selbst wenn natürlich richtig ist, dass die Ziele und normativen Grundlagen von GCE auch in ,Westlichen Traditionen' verankert sind, bedeutet dies weder, dass sie nicht auf andere Kontexte angewendet werden bzw. in diesen adaptiert werden könnten, noch dass sie per se als illegitim, eurozentristisch oder neo-imperialistisch abzuqualifizieren sind (Culp, 2019). Historische Kritiken von Kolonialismus und Imperialismus basierten im Gegenteil auf universellen Werten und Prinzipien (wie den Menschenrechten), so wie auch prominente Kritiker des Kolonialismus (wie etwa Tagore) häufig Verteidiger universalistischer und kosmopolitischer Ideale waren (z. B. Mishra, 2013). Es sollte daher nicht vergessen werden, dass "the denial of Enlightenment ideals, not their assertion, characterised colonialism and imperialism" (Butcher, 2018, S. 20).

Während natürlich immer die Gefahr besteht, dass eine universalistische Konzeption von GCE auf eine Weise genutzt wird, die „ethnocentric, ahistorical, depoliticized, paternalistic, salvationist and triumphalist" (Andreotti \& de Souza, 2012, S. 1) ist, und man zugleich auch aus historischen Gründen hinreichend Anlass hat, skeptisch zu sein bezüglich jeder Form eines universalistischen westlichen Triumphalismus (z. B. im Namen der Menschenrechte: Joas, 2015) oder bestimmter Vorstellungen von Fortschritt und Entwicklung (Allen, 2017), bedeutet dies nicht, dass vom potentiellen Missbrauch von pädagogischen und politischen Idealen auf deren intrinsische Illegitimität zu schließen ist. Es ist z. B. natürlich sinnvoll und vernünftig, fragwürdige Nutzungen von Mills oder Lockes Konzeptionen der Autonomie und Rationalität (Arneil, 2012) oder rassistische Elemente in Kants und Hegels Konzeptionen der Bildung (Wischmann, 2018) zu kritisieren. Von diesen historischen Beispielen bzw. auch von historischen Entstehungsund Verwendungskontexten eines Ideals und einer Idee zu schließen, dass die Ideen der Autonomie, der Rationalität oder der Bildung per se und essentiell kontaminiert wären mit kolonialen Herrschaftsansprüchen, ist ein Non-sequitur und läuft auf normativistische Formen der historischen Dekontextualisierung hinaus. Die Ambivalenz von nahezu allen pädago- gischen und politischen Idealen beruht darauf, dass sie in der Regel auch benutzt werden können, illegitime Formen der Herrschaft zu rechtfertigen, dass sie aber auch zur Rechtfertigung von legitimen Ansprüchen auf Emanzipation herangezogen werden können. Diese Ambivalenz zu ignorieren läuft auf eine moralistisch-dogmatische Form des Essentialismus hinaus.

Darüber hinaus ist es problematisch, dass postkoloniale Kritiken in vielen Fällen die normativen Grundlagen ihrer Kritiken nicht hinreichend offenlegen, was nicht zuletzt darauf zurückzuführen sein mag, dass sie häufig starke Anleihen bei älteren Versionen der kritischen Theorie und poststrukturalistischen und postmodernen Ansätzen machen. ${ }^{10}$ So wird sehr viel Aufwand betrieben, um westliche Ideale zu kritisieren, ohne dass man jedoch pädagogische und politische Alternativkonzeptionen (zu den ,westlichen') ausarbeitet und begründet, die in einer zunehmend integrierten und globalisierten Welt praktikabel wären. Andreotti argumentiert z. B., dass pädagogische Interaktionen im Rahmen von GCE sich orientieren sollten an „radical appeals to openness, to difference and to the negotiation of meaning, rather than around normative appeals to notions of impartial reasoning or ideas of democracy, freedom, rights and justice that are presented as universal" (Andreotti, 2010, S. 234). Es scheint mir jedoch, dass die Konstruktion von Dichotomien zwischen diesen Typen von Zielen nicht nur etwas artifiziell anmutet, sondern auch, dass die von Andreotti propagierten alternativen Ziele extrem vage und unklar bleiben, insbesondere mit Bezug auf pädagogische Konstellationen, die nun einmal asymmetrisch strukturiert sind (hierzu auch: Bechtum \& Overwien, 2017).

Diese Kritiken laufen in jedem Fall Gefahr (auch wenn das häufig nicht intendiert ist), relativistische Argumente für fragwürdige Formen des Partikularismus und Tribalismus zu liefern (so wie sie z. B. in Diktaturen propagiert werden) und zugleich das emanzipatorische Potential von unterschiedlichen Formen des Universalismus zu ignorieren (Wright, 2012). Es bleibt z. B. unklar, warum der Versuch, Kinder dazu in die Lage zu versetzen, ihre Rechte wahrzunehmen, als Perpetuierung westlicher Dominanz qualifiziert werden sollte. Genauso bleibt unklar, wie eine Konzeption der „hyper-self-reflexivity as a strategy that acknowledges everyone's complicities and investments in coercive and repressive belief systems" (Andreotti, 2010, S. 238), die von postkolonialen Kritikerinnen verteidigt wird, gerechtfertigt werden könnte, ohne auf, liberale‘ Konzeptionen des Selbst oder der Autonomie zurückzugreifen, die man doch immer schon vorauszusetzen scheint, wenn man diese Form der Kritik propagiert. Wie sollte man zudem historisch gewachsene globale Ungerechtigkeiten gänzlich ohne Rekurs auf universalistische Geltungsansprüche kritisieren? Im Rahmen einer relativistischen Eingrenzung der Geltung z. B. der Menschenrechte auf eine bestimmte ,Kultur' wird dies mit Sicherheit nicht gelingen (vgl. auch die Kritiken von: Höffe, 2008 und Krämer, 2013; sowie auch: Appiah, 1991).

Die postkoloniale Kritik liegt jedoch richtig mit der Annahme, dass es für Verteidiger von GCE von zentraler Wichtigkeit ist, die partikulare Einbettung ihrer universalistischen Ambitionen kritisch zu reflektieren und zu hinterfragen (Dill, 2015) und zugleich fragwürdige Formen des Missbrauchs von Universalismen oder Pseudo-Universalismen zu einem zentra- 
len Thema und Problem von GCE zu machen. Universalismus kann in seinen unterschiedlichen Spielarten in unterschiedlichen Traditionen verwurzelt sein und die Anwendung universeller Werte und Prinzipien kann und sollte nicht im Sinne einer simplen ,exercise in deduction' verstanden werden. Dies und auch das Faktum, dass es natürlich unterschiedliche Wege der GCE im Rekurs auf unterschiedliche lokale Traditionen geben kann (Mouffe, 2015; Wohlfahrt, 2018), impliziert jedoch nicht, dass wir auf universalistische moralische, politische und pädagogische Geltungsansprüche im Kontext von GCE verzichten bzw. diese aufgeben könnten. Dies betrifft auch Vertreter postkolonialer Theorie, die in rechtfertigungstheoretische Schwierigkeiten geraten, wenn sie für ihre Kritiken nicht (in welcher Form auch immer) auf universalistische Vorgaben zurückzugreifen können (wie z. B. die Menschenrechte oder personale Autonomie). Die Annahme z. B., dass ein Oktroi von Wertvorstellungen auf andere gesellschaftliche oder kulturelle Kontexte moralisch inakzeptabel ist, und die Überzeugung, dass jede Person und jede kulturelle Orientierung Respekt verdient, scheint offenkundig selbst ein transkulturelles und universalistisches Ideal zu sein. Dieses universalistische Ideal wird schließlich auch von Kritikern des, Universalismus' verteidigt und zwar unabhängig von spezifischen kulturellen Einbettungen - man wendet die entsprechenden normativen Vorgaben vielmehr auch auf solche Gruppen an, die sie ggf. nicht unbedingt teilen. Letztlich kann man sich in einigen Fällen des Eindrucks nicht erwehren, dass einige der postkolonialen Kritiken von GCE eher anachronistisch wirken, wenn sie von geopolitischen Annahmen über internationale Herrschaftsstrukturen ausgehen, welche im 19ten und 20ten Jahrhundert weitgehend zutrafen, jedoch nicht mehr ohne weiteres auf die Situation im 21ten Jahrhundert angewendet werden können (Baldwin, 2016). Dies ist einer der Gründe dafür, dass GCE in Zukunft auf eine wirklich universelle Kritik von unterschiedlichen historischen und aktuellen Formen des (Neo-)Imperialismus und (Neo-)Kolonialismus ausgerichtet sein sollte, und zwar überall auf der Welt und nicht nur mit Bezug auf den ,Westen'.

Das zentrale Problem und die zentrale Herausforderung bestehen heute weniger darin, das Partikulare vor dem Universellen zu schützen, sondern primär darin, das Universelle vor dem Partikularen zu schützen. In einer zunehmend global integrierten Welt, in welcher unterschiedliche Formen des moralischen und politischen Partikularismus auf dem Vormarsch sind (z. B. autoritäre Formen des Populismus und Fundamentalismus: hierzu, Oelkers, 2018), bedarf es der auf Integration, nicht der auf Separation und Besonderung angelegten pädagogischen Ideale und politischen Orientierungen. Edward Said hat hierzu treffend festgestellt: "What does need to be remembered is that narratives of emancipation and enlightenment in their strongest form were also narratives of integration not separation, the stories of people who had been excluded from the main group, but who were now fighting for a place in it" (Said, 1994, S. xxxiii).

Man kann daher davon ausgehen, dass universalistische pädagogische und politische Ideale weder durch ihre Entstehungsbedingungen normativ fixiert sind, noch durch die immer gegebene Möglichkeit ihres Missbrauchs per se illegitim werden. Zudem können auch postkoloniale Kritiken von glo- balen pädagogischen und politischen Ungerechtigkeiten auf universalistische Geltungsansprüche nicht verzichten. Zugleich sollte auch die postkoloniale Kritik an eurozentristischen Orientierungen von Konzeptionen der GCE selbst Bestandteil einer (selbst-)kritischen und universalistischen Konzeption des GCE sein.

\section{Fazit}

In diesem Beitrag habe ich eine universalistische Konzeption der GCE gegen einen ihrer wichtigsten Kritiken verteidigt. Der Vorwurf, GCE basiere auf genuin westlichen Werten und sei deshalb bloß eine weitere Manifestation einer pädagogischen Rechtfertigung von Imperialismus und Kolonialismus, stützt sich auf fragwürdige theoretische, normative und empirische Prämissen, die sich nicht aufrecht erhalten lassen. Zugleich stimme ich mit Andreotti (2010) und anderen Vertretern postkolonialer Theorie überein, dass eine plausible und historisch informierte Konzeption der GCE zentrale Elemente von postkolonialen Kritiken berücksichtigen muss. Ich hoffe, so einige überzeugende Gründe geliefert haben für die Annahme, dass die Leitprinzipien einer (selbst-)kritischen Konzeption der GCE sich nicht angemessen ohne Rekurs auf universalistische Geltungsansprüche begründen lassen.

\section{Anmerkungen}

1 Einen historischen Überblick über ideologische Rechtfertigungen des britischen Empires bietet: Pitts (2005); Für den Fall der deutschen Kolonien: Gründer \& Hiery (2018). Die folgenden Überlegungen stellen eine revidierte und erweiterte Version des dritten Unterkapitels aus Drerup (2020) dar.

2 Eine Möglichkeit das Verhältnis und die Differenz von Kolonialismus und Imperialismus zu verstehen, geht davon aus, dass Imperialismus „can function without formal colonies (as in United States imperialism today) but colonialism cannot" (Loomba, 2015, S. 28); für eine andere Diskussion der Unterscheidung vgl.: Castro Varela \& Dhawan (2015).

3 Nach Andreotti geht es postkolonialer Theorie vor allem um „the epistemic violence of colonialism and the interrogation of European cultural supremacy in the subjugation of different peoples and knowledges in colonial and neocolonial contexts" (Andreotti, 2010, S. 238). Loomba versteht die ,postcolonial studies' nicht als ,tightly bound 'field' as such" (Loomba, 2015, S. 3) und verweist auf die Problematik einer Überdehnung des Konzepts des Postkolonialen (ebd., S. 34). Im Folgenden werde ich die Termini ,postkolonial' und ,dekolonial' nicht unterscheiden, da es eher unklar bleibt, ob und inwieweit es eine reale und relevante Differenz zwischen beiden Ansätzen gibt. Dekoloniale Theoretiker kritisieren postkoloniale Theoretiker unter anderem dafür, dass deren Kritik der ,westlichen Epistemologie selbst auf einer ,westlichen' und eurozentristischen Epistemologie basiert (Castro Varela \& Dhawan 2015, S. 320). Mir ist entsprechend bewusst, dass das Feld der postkolonialen Studien (wie auch der GCE) eine immense Diversität von Ansätzen umfasst. Ich beanspruche daher nicht, dass meine Kritik der Kritik auf alle postkolonialen Ansätze in gleicher Weise zutrifft.

4 Der Begriff, Global Citizenship Education` kann als funktional und semantisches Äquivalent zu dem im deutschsprachigen Raum gebräuchlicheren Begriff des ,globalen Lernens' verstanden werden (hierzu: Asbrand \& Scheunpflug, 2014).

5 Mir sind die Schwierigkeiten der Operationalisierung dieser Konzepte im Rahmen z. B. von kompetenztheoretischen Ansätzen durchaus bewusst: vgl. Asbrand \& Scheunpflug (2014); Sälzer \& Roczen (2018). Auch wenn diese Schwierigkeiten zum Teil in verschiedenen, teilweise konfligierenden Konzeptionen der globalen Gerechtigkeit wurzeln, die von transnationalen Organisationen propagiert werden, bedeutet dies weder, dass dies zu unüberwindbaren Begründungshindernissen für eine normative Konzeption der GCE führt, noch dass eine solche Konzeption notwendig im Kontext eines kompetenztheoretischen Rahmens konkretisiert werden muss.

6 Zur Rolle des Internets für die Emergenz und Etablierung einer globalen Öffentlichkeit und Demokratie: Wegerif (2018). 
7 Vgl. zu diesem Thema der Beitrag von Kai Horsthemke in diesem Themenheft. Vgl. auch Castro Varela \& Heinemann (2016).

8 Dieses Problem wird erstaunlich häufig übersehen (für eine Ausnahme: Asbrand \& Scheunpflug, 2014, S. 405; Overwien, 2016) in Konzeptionen der Bildung und Erziehung, die sich starken Zielen sozialer und globaler Gerechtigkeit verschrieben haben (Yacek, 2017; zum Konzept der Indoktrination: Drerup, 2018). Dennoch denke ich, dass Kernaspekte der hier vertretenen Konzeption von GCE gegen entsprechende Vorbehalte verteidigt werden können (insbesondere auf Grund des starken Fokus auf personale und politische Autonomie).

9 Said hat entsprechend treffend festgestellt: „The fact is, we are mixed in with one another in ways that most national systems of education have not dreamed of "(Said, 1994, S. 426).

10 Hierauf zu verweisen läuft m. E. nicht automatisch auf die unkritische Reproduktion eurozentristischer Superioritätsvorstellungen hinaus. Wenn man bestimmte Theorien importiert, muss man eben ein Stück weit damit leben bzw. sich damit auseinandersetzen, dass man sich so in der Regel zugleich auch auf problematische und kritikwürdige methodologische und epistemologische Prämissen festlegt - die es offenzulegen und zu diskutieren gilt.

\section{Literatur}

Allen, A. (2017). Adorno, Foucault, and the End of Progress: Critical Theory in Postcolonial Times. In P. Deutscher \& C. Lafont (Hrsg.), Critical Theory in Critical Times (S. 183-207). New York: Columbia University Press. https://doi.org/10.7312/ columbia/9780231181518.003.0009

Andreotti, V. (2010). Postcolonial and post-critical global citizenship education. In G. Elliot, C. Fourali \& S. Issler (Hrsg.), Education and social change: Connecting local and global perspectives (S. 233-245), London: Continuum International.

Andreotti, V. \& de Souza, L. (2012). Introduction: (Towards) Global Citizenship Education 'Otherwise'. In V. Andreotti \& L.de Souza (Hrsg.), Postcolonial Perspectives on Global Citizenship Education (S. 1-6), New York \& London: Routledge. https://doi.org/10.4324/9780203156155

Appiah, A.K. (1991). Is the Post- in Postmodernism the Post- in Postcolonial? Critical Inquiry, 17(2), 336-357.

Appiah, A. K. (2018). The lies that bind. Rethinking identity. London: Profile Books. Arneil, B. (2012). Liberal Colonialism, Domestic Colonies and Citizenship. History of Political Thought, 23, 491-523.

Asbrand, B. \& Scheunpflug, A. (2014). Globales Lernen. In W. Sander (Hrsg.), Handbuch Politische Bildung (S. 401-414), Schwalbach: ZpB.

Baldwin, R. (2016). The Great Convergence. Cambridge, MA, London: Harvard University Press.

Barnett, M. (2017). Conclusion: The World According to Paternalism. In M. Barnett (Hrsg.), Paternalism without Borders (S. 316-344). Cambridge: Cambridge University Press. https://doi.org/10.1017/9781316799956.011

Bechtum, A. \& Overwien, B. (2017). Kann postkoloniale Kritik Schule machen? Über ihre Grenzen und Potenziale für (entwicklungs-)politische Bildungsarbeit. In H.-J. Burchardt, S. Peters \& N. Weinmann (Hrsg.), Entwicklungstheorie von heute - Entwicklungspolitik von morgen, (S. 59-85). Baden-Baden: Nomos. https://doi. org/10.5771/9783845267340-60

Brumlik, M. (2016). Kosmopolitische Moral im Zeitalter der Globalisierung: Menschenrechtliche Bildung und globale Erinnerungskultur. In S. Weyer \& N. Köb (Hrsg.), Bildung und Menschenrechte (S. 69-89). Wiesbaden: Springer VS. https:// doi.org/10.1007/978-3-658-11687-3_5

Butcher, J. (2018). Questioning the Epistemology of Decolonise: The Case of Geography. Social Epistemology Review and Reply Collective, 7(11), 12-24.

Castro Varela, M. \& Dhawan, N. (2015). Postkoloniale Theorie. Bielefeld: transcript. https://doi.org/10.14361/9783839411483

Castro Varela, M. \& Heinemann, A. B. (2016). Globale Bildungsbewegungen Wissensproduktionen verändern. Zeitschrift für internationale Bildungsforschung und Entwicklungspädagogik, 39(2), 17-22.

Chandra-Milena, D. (2013): Überlegenheitsdenken fällt nicht vom Himmel. Postkoloniale Perspektiven Globales Lernen und Bildung für nachhaltige Entwicklung. Zeitschrift für internationale Bildungsforschung und Entwicklungspädagogik, 36(1), 26-33.

Conrad, S. (2016). What is Global History? Princeton: Princeton University Press.

Culp, J. (2018). Internationalizing Nussbaum's model of cosmopolitan democratic education, Ethics and Education, 13(2), 172-190. https://doi.org/10.1080/174496 42.2018.1439308

Culp, J. (2019). Democratic Education in a Globalized World. New York \& Oxon: Routledge. https://doi.org/10.4324/9780367136550 de Ruyter, D. \& Spiecker, B. (2008). The World Citizen Travels with a Different View. In M. Peters, A. Britton \& H. Blee (Hrsg.), Global Citizenship Education (S. 351-364). Rotterdam \& Taipei: Sense publishers. https://doi.org/10.1163/9 789087903756_023

Dill, J. (2015). The Longings and Limits of Global Citizenship Education. New York: Routledge.

Drerup, J. (2018). „Zwei und zwei macht vier.“ Über Indoktrination und Erziehung. Diskurs Kindheits- und Jugendforschung, 1, 7-24. https://doi.org/10.3224/diskurs.v13i1.02

Drerup, J. (2020). Global Citizenship Education, Global Educational Injustice and the Postcolonial Critique. Erscheint in Global Justice. Theory Practice Rhetoric.

Dübgen, F. (2017). Fortschritt im Widerstreit - Dekolonisierung als Kritik? Deutsche Zeitschrift für Philosophie, 65(1), 163-173. https://doi.org/10.1515/dzph2017 $-001$

Enslin, P. \& Horsthemke, K. (2015). Rethinking the Western Tradition. Educational Philosophy and Theory, 47(11), 1166-1174. https://doi.org/10.1080/00131857.20 14.991501

Fukuyama, F. (2018). Identity Politics. London: Profile Books.

Gaudelli, W. (2016). Global Citizenship Education, Everyday Transcendence. Oxon $\&$ New York: Routledge.

Gründer, H. \& Hiery, H. (Hrsg.) (2018). Die Deutschen und ihre Kolonien. Berlin $\mathrm{ZpB}$.

Hall, S. (1992). The West and the rest: Discourse and power. In S. Hall \& B. Gieben (Hrsg.), Formations of Modernity (S. 275-331). Cambridge: Polity Press.

Held, D. (1995). Democracy and the Global Order. Stanford: Stanford University Press.

Hendriks, A. (2018). Tomaten. Bonn: Bundeszentrale für politische Bildung.

Hobson, J. (2017). Eurocentric Pitfalls and Paradoxes of International Paternalism: Decolonizing Liberal Humanitarianism 2.0. In M. Barnett (Hrsg.), Paternalism without Borders (S. 99-131). Cambridge: Cambridge University Press. https://doi. org/10.1017/9781316799956.004

Höffe, O. (2008). Koexistenz der Kulturen im Zeitalter der Globalisierung. München: Münchner Kompetenzzentrum Ethik. https://doi.org/10.14361/9783839407 684005

Joas, H. (2015). Sind die Menschenrechte westlich? München: Kösel.

Krämer, G. (2013). Von antirassistischen Denkverboten. Anmerkungen zur Rassismus-Debatte in der entwicklungspolitischen Bildung. Zeitschrift für internationale Bildungsforschung und Entwicklungspädagogik, 36(3), 32-34.

Lee, C. (2014). Decolonizing global citizenship. In E. Isin \& P. Nyers (Hrsg.), Routledge Handbook of Global Citizenship Studies (S. 75-85). London \& New York: Routledge.

Liebel, M. (2017). Postkoloniale Kindheiten. Weinheim: Beltz

Loomba, A. (2015). Colonialism/Postcolonialism. London \& New York: Routledge. https://doi.org/10.4324/9781315751245

Malik, K. (2014). The Quest for a Moral Compass. London: Atlantic Books.

McCarthy, T. (2009). Race, Empire, and the Idea of Human Development. Cambridge: Cambridge University Press. https://doi.org/10.1017/CBO9780511814044 Mignolo, W. (2009). Who speaks for the 'Human' in Human Rights? Hispanic Issues On Line, 5(1), 7-24.

Mishra, P. (2013). From the Ruins of Empire. London et al.: Penguin.

Mouffe, C. (2015). Agonistik. Bonn: bpb.

Nederman, C. (2012). Toleration in a new key: historical and global perspectives. In D. Edyvane \& M. Matravers (Hrsg.), Toleration Re-Examined (S. 69-82). London \& New York: Routledge.

Oelkers, J. (2018). Autoritarismus und liberale öffentliche Bildung. Zeitschrift für Pädagogik, 64(6), 728-748.

Overwien, B. (2016). Globales Lernen und politische Bildung - eine schwierige Beziehung? Zeitschrift für internationale Bildungsforschung und Entwicklungspädagogik, 39(2), 7-11.

Oxley, L. \& Morris, P. (2013). Global Citizenship: A Typology for Distinguishing its Multiple Conceptions. British Journal of Educational Studies, 61(3), 301-325. https://doi.org/10.1080/00071005.2013.798393

Pitts, J. (2005). A Turn to Empire. Princeton. Oxford: Princeton University Press. https://doi.org/10.1515/9781400826636

Reinhard, W. (2018). Die Unterwerfung der Welt. Bonn: BpB.

Said, E. (1994). Culture \& Imperialism. New York: Vintage Books.

Sälzer, C. \& Roczen, N. (2018). Die Messung von Global Competence im Rahmen von PISA 2018. Zeitschrift für Erziehungswissenschaft, 21, 299-316. https://doi. org/10.1007/s11618-018-0818-y 
Sandel, M. (1998). Liberalism and the Limits of Justice. Cambridge: Cambridge University Press. https://doi.org/10.1017/CBO9780511810152

Sant, E., Davies, I., Pashby, K. \& Shultz, L. (2018). Global Citizenship Education. London: Bloomsbury.

Scheunpflug, A. (2014). Globales Lernen und die Debatte über Postkolonialität. Zeitschrift für internationale Bildungsforschung und Entwicklungspädagogik, 37(4), 31-32.

Sen, A. (2005). The Argumentative Indian. New York: Farrar, Straus and Giroux. Sen, A. (2006). Identity and Violence. London: Penguin.

Taylor, C. (1989). Sources of the Self. Cambridge, MA: Harvard University Press.

Tully, J. (2008). Two Meanings of Global Citizenship Education: Modern and Diverse. In M. Peters, A. Britton \& H. Blee (Hrsg.), Global Citizenship Education (S. 15-40). Rotterdam \& Taipei: Sense publishers. https://doi.org/10.1163/978 $9087903756 \_003$

Wegerif, R. (2018). New Technology and the Apparent Failure of Democracy: An Educational Response. On Education. Journal for Research and Debate, 1(1).

Weidner, S. (2018). Jenseits des Westens. Bonn: bpb.

Wischmann, A. (2018). The absence of 'race' in German discourses on Bildung. Rethinking Bildung with critical race theory. Race, Ethnicity and Education, 21(4), 471-485. https://doi.org/10.1080/13613324.2016.1248834
Wohlfahrt, G. (2018). Global Citizenship Education (GCED) in Myanmar. Am Beispiel eines Projekts der internationalen Bildungszusammenarbeit. Zeitschrift für internationale Bildungsforschung und Entwicklungspädagogik, 41(1), 23-28.

Wright, C. (2012). Postcolonial Cosmopolitanisms: Towards a Global Citizenship Education based on 'Divisive Universalism'. In V. Andreotti \& L. de Souza (Hrsg.), Postcolonial Perspectives on Global Citizenship Education (S. 47-67). New York \& London: Routledge.

Yacek, D. (2017). Transformation and Education. A Philosophical Inquiry. Columbus, OH: Ohio Link.

\section{Dr. Johannes Drerup}

ist Professor für Allgemeine Erziehungswissenschaft mit dem Schwerpunkt Bildungstheorie an der TU Dortmund sowie Gastprofessor an der Vrije Universiteit Amsterdam. Seine Forschungsinteressen beinhalten die Themenschwerpunkte Philosophy of Education, Allgemeine Erziehungswissenschaft/Erziehungs- und Bildungstheorie, Philosophie der Kindheit, politische Philosophie der Erziehung und Bildung sowie pädagogische Ethik/angewandte Ethik.

\section{UNSERE BUCHEMPFEHLUNG}



2019, 256 Seiten, br., 34,90€, ISBN 978-3-8309-3940-5

E-Book: Open Access ISBN 978-3-8309-8940-0
Erwin Rauscher, Christian Wiesner, Daniel Paasch, Petra Heißenberger (Hrsg.)

\section{Schulautonomie - Perspektiven in Europa Befunde aus dem EU-Projekt INNOVITAS}

Schulautonomie erscheint gegenwärtig sowohl als Hoffnungswort wie auch als eine Art Zauberformel für die Schulentwicklung in vielen europäischen Ländern. Die Entwicklungen in den einzelnen Ländern unterscheiden sich dennoch sehr - es gilt, mit- und voneinander zu lernen: Wie unterschiedlich funktioniert Schulautonomie im Spannungsverhältnis von Freiheit am Schulstandort und Rechenschaftspflicht gegenüber den zuständigen Behörden? In den Beiträgen des Bandes wird der europäische Diskurs um Schulautonomie und ihre Auswirkungen aus verschiedenen Perspektiven in den Ländern und Regionen - juristisch, national und international vergleichend - beleuchtet. 